\title{
BMJ Open Factors associated with health literacy in multimorbid patients in primary care: a cross-sectional study in Switzerland
}

\author{
Alexandra A N'Goran, ${ }^{1}$ Jérôme Pasquier, ${ }^{2}$ Anouk Deruaz-Luyet, ${ }^{1}$ Bernard Burnand, ${ }^{2}$ \\ Dagmar M Haller, ${ }^{3}$ Stefan Neuner-Jehle, ${ }^{4}$ Andreas Zeller, ${ }^{5}$ Sven Streit, ${ }^{6}$ Lilli Herzig, ${ }^{1}$ \\ Patrick Bodenmann ${ }^{7}$
}

To cite: N'Goran AA, Pasquier J, Deruaz-Luyet A, et al. Factors associated with health literacy in multimorbid patients in primary care: a cross-sectional study in Switzerland. BMJ Open 2018;8:e018281. doi:10.1136/ bmjopen-2017-018281

- Prepublication history for this paper is available online. To view these files, please visit the journal online (http://dx.doi. org/10.1136/bmjopen-2017018281).

$\mathrm{LH}$ and PB contributed equally.

Received 26 June 2017 Revised 25 December 2017 Accepted 3 January 2018

Check for updates

${ }^{1}$ Institute of Family Medicine, University of Lausanne, Lausanne, Switzerland ${ }^{2}$ Institute of Social and Preventive Medicine, Lausanne University Hospital, Lausanne, Switzerland

${ }^{3}$ Primary Care Unit, Faculty of Medicine, University of Geneva, Geneva, Switzerland

${ }^{4}$ Institute of Primary Care, University of Zurich, Zürich, Switzerland

${ }^{5}$ Centre for Primary Health Care, University of Basel, Basel, Switzerland

${ }^{6}$ Institute of Primary Health Care (BIHAM), University of Bern, Bern, Switzerland

${ }^{7}$ Department of Ambulatory Care and Community Medicine, Lausanne University Hospital, Lausanne, Switzerland

Correspondence to

Dr Lilli Herzig; lilli.herzig@hin.ch

\section{ABSTRACT}

Objective To identify factors associated with health literacy in multimorbid patients.

Design A nationwide cross-sectional study in Switzerland. Univariate and multivariate linear regressions were calculated to identify variables associated with health literacy. A multiple imputation approach was used to deal with missing values.

Participants Multimorbid patients recruited in primary care settings $(n=888)$, above 18 years old and suffering from at least 3 of 75 chronic conditions on a predefined list based on the International Classification of Primary Care 2. Main measures Health literacy was assessed using the European Health Literacy Survey project questionnaire (HLS-EU 6). This comprises six items scored from 1 to 4 (very difficult $=1$, fairly difficult $=2$, fairly easy $=3$, very easy=4), and the total health literacy score is computed as their mean. As we wished to understand the determinants associated with lower health literacy, the HLS-EU 6 score was the only dependent variable; all other covariates were considered independent.

Results The mean health literacy score (SD) was 2.9 (0.5). Multivariate analyses found significant associations between low health literacy scores and treatment burden scores ( $\beta=-0.004,95 \% \mathrm{Cl}-0.006$ to 0.002$)$; marital status, predominantly the divorced group $(\beta=0.136$, $95 \% \mathrm{Cl} 0.012$ to 0.260 ); dimensions of the EuroQuol 5 Dimension 3 Level (EQ5D3L) quality of life assessment, that is, for moderate problems with mobility $(\beta=-0.086$, $95 \% \mathrm{Cl}-0.157$ to 0.016 ); and with moderate problems ( $\beta=-0.129,95 \% \mathrm{Cl}-0.198$ to 0.060$)$ and severe problems with anxiety/depression $(\beta=-0.343,95 \% \mathrm{Cl}-0.500$ to 0.186).

Conclusions Multimorbid patients with a high treatment burden, altered quality of life by problems with mobility, anxiety or depression, often also have low levels of health literacy. Primary care practitioners should therefore pay particular attention to these patients in their daily practice.

\section{INTRODUCTION}

Multimorbidity, defined as the occurrence of multiple chronic medical conditions in one individual, ${ }^{1-3}$ is a steadily increasing phenomenon due to population ageing. ${ }^{45}$ Multimorbid patients must face
Strengths and limitations of this study

This national primary care study enabled the analysis of data from a sample of multimorbid patients.

- The first study to understand factors associated with health literacy in multimorbid patients in primary care settings.

- Only multimorbid patients with at least three chronic conditions were assessed.

- Causal relationships could not be inferred due to the study design.

many challenges: more frequent and longer hospitalisations, ${ }^{6} 7$ greater use of polypharmacy (causing adverse drug effects), 89 higher expenditure on healthcare ${ }^{10-12}$ and the use of a broader range of healthcare services. $^{810}$ Moreover, as the number of health professionals involved in treatment increases, the more likely patients will be faced with fragmented medical care due to conflicting instructions and care pathways. This makes piecing together and adhering to instructions even more testing and thus prevents patients from participating effectively in their own care. ${ }^{8} 1012$ Facing all these challenges effectively requires good levels of health literacy (HL). The US Institute of Medicine defines HL as 'the degree to which individuals have the capacity to obtain, process and understand basic health information and services needed to make appropriate health decisions. ${ }^{13} 14 \mathrm{HL}$ includes a broad set of skills (ie, reading, writing, numeracy, communication and increasingly, the use of digital technologies) needed to make appropriate health decisions and successfully navigate the healthcare system. ${ }^{15} \mathrm{HL}$ is recognised as an important determinant of health. ${ }^{16-18}$ Studies have shown that lower HL is associated with a lower mental and physical health status, adverse disease-specific 
outcomes, higher mortality and more use of healthcare but less use of preventive care. ${ }^{17}{ }^{19}$ Consequently, governments, researchers, clinicians and patients' associations are paying ever more attention to research into HL. ${ }^{20}$

Effective patient-clinician communication that ensures patients are able to understand the health information and treatment recommendations they receive and feel comfortable enough to ask questions or admit when they do not understand something, is vital to the successful management of a chronic illness. ${ }^{21}$ Healthcare providers should be conscious of their patients' HL skills so as to ensure that health information is communicated effectively to help manage long-term conditions. ${ }^{22} 23$ Additionally, HL is a prerequisite for patient activation and shared decision-making. ${ }^{24-26}$ Thus, identifying factors associated with low HL is an important step towards devising effective engagement, prevention and intervention strategies for patients in primary care. ${ }^{20}$ The literature shows that HL has been assessed in different ways and with contrasting conclusions, for example, different studies looking at the factors influencing HL among less well-educated young people showed a relationship between low HL and socioeconomic factors. ${ }^{172728}$ One study evaluating relevant associations between HL and multimorbidity (defined as two or more chronic diseases from a list of 11 conditions) in primary care, found none. ${ }^{14}$ To better understand the determinants associated with low HL, the present study aimed to explore all the factors that might be associated with low HL in multimorbid patients in primary care with at least three chronic conditions.

\section{METHODS}

\section{Participants and procedures}

We analysed data $(\mathrm{n}=888)$ from a national cross-sectional survey conducted in collaboration with Switzerland's five academic institutes of family medicine, between January and September 2015. The study was designed to assess multimorbidity in patients in a primary care setting in order to target a population whose management is more challenging to general practitioners (GPs). The detailed study protocol, dataset description and initial results have been published elsewhere. ${ }^{29} 30$

A convenience sample of 100 GPs randomly enrolled patients from their practices who consulted them during the study period. Each GP was provided with a randomisation calendar specifying which patients to enrol on each half-day during the recruitment weeks. All multimorbid patients above 18 years old and suffering from at least 3 of 75 chronic conditions on a predefined list, based on the International Classification of Primary Care 2 (ICPC-2), were considered eligible ${ }^{31} 32$ and gave written informed consent to participate in the study. GPs completed a paper-based questionnaire for each included patient (patient-related variables assessed through the GP survey). Patients enrolled completed a telephone-based questionnaire.

\section{Measures}

Health literacy

HL was assessed using the validated six-item questionnaire from the European Health Literacy Survey project (HLS-EU 6). ${ }^{33}{ }^{34}$ The HLS-EU was a Europe-wide project developed to gather data on HL. ${ }^{33}$ The original HLS-EU 47 explored three domains: (1) healthcare; (2) disease prevention and (3) health promotion. Each domain explored four matrices: accessing/obtaining information; understanding information; processing/appraising information and applying/using information. The HLS-EU 6 is a validated short form with two of the original questions remaining in each domain. ${ }^{33-35}$ Validated French and German versions of the HLS-EU 6 were available by the authors. ${ }^{34}$ We used this shorter, validated questionnaire because the present study's main objective was to measure overall levels of HL in multimorbid patients, not to assess HL in detail. The HLS-EU 6 scale consists of six items with five possible responses. Participants were asked to respond to the following questions. How easy or difficult is it for you to: (1) judge when you may need to get a second opinion from another doctor? (2) use information the doctor gives you to make decisions about your illness? (3) find information on how to manage mental health problems like stress or depression? (4) judge whether the information on health risks in the media is reliable? (5) find out about activities that are good for your mental well-being? and (6) understand information in the media on how to get healthier? The possible responses and their scores were as follows: very difficult $=1$, fairly difficult $=2$, fairly easy $=3$, very easy $=4$, and a fifth alternative for when participants did not answer or did not have a definite answer, coded as a missing value. The HL score was thus calculated as the mean of the six HL items, scored from 1 to 4 , after imputation of the missing values (see Statistical analyses section). ${ }^{34}$

\section{Covariates}

All the variables have been described elsewhere previously. ${ }^{29}$ Briefly, our analyses used the following variables:

- Dependent variable: as we wished to understand the determinants associated with lower HL scores, we chose the HLS-EU as the dependent variable.

- Independent variables: included all other variables (based on GP and patient questionnaires):

- Age

- Sex (female/male).

GP's questionnaire (based on his medical records):

- Number of chronic conditions based on a predefined list of 75 chronic conditions relevant to multimorbidity and coded according to the ICPC 2;

- Number of drugs $(0-4,5-6,7-9$ or $\geq 10)$ listed by organic system (general, blood, immune, digestive, cardiovascular, respiratory, neurological, 
endocrine, eye, ear, psychological, musculoskeletal) and by substance;

- Number of medical visits in the last year;

- Number of hospitalisations in the last year;

- The Severity Index (SI) ${ }^{36-38}$ was derived by dividing the total Cumulative Illness Rating Scale score, a validated questionnaire published elsewhere in detail, ${ }^{39}$ by the number of categories with morbidities.

Patient interview (based on a questionnaire):

- Marital status (single, married, divorced or widowed);

- Educational level (primary, secondary and tertiary);

- DipCare questionnaire for deprivation assessment, containing 16 items examining the three dimensions of deprivation (material, social and health), and validated for the Swiss population and published elsewhere in detail $;^{40}$

- Treatment Burden Questionnaire, recently developed to produce a score for the overall burden related to the treatment of chronic conditions ${ }^{41}$; it consists of 13 questions to rate with an answer between 0 and 10 , with 0 corresponding to no burden and 10 to a very important/considerable burden;

- Medical help from a home nurse;

- Paramedical therapist grouped together physiotherapist and/or occupational therapist (yes/no);

- Homecare (yes/no);

- Number of specialists involved in patient's care;

- EuroQuol 5 Dimension 3 Level (EQ5D3L) composed of five dimensions of health (mobility, selfcare, usual activities, pain/discomfort and anxiety/ depression) and used a Visual Analogue Scale.

\section{Statistical analyses}

On collecting the questionnaires, the number of missing values from our variable of interest (HL score) was considered too high (ie, the HL score could not be computed in full for 577 participants) to reasonably analyse each participant's case in its entirety. We first considered computing the HL scores for all participants with at least five non-missing items. However, even then, the number of missing scores remained too high (377 participants), and comparisons between complete and the incomplete sets of responses showed significant differences in several covariates (data not shown). We therefore opted for the imputation of the missing values from the HL items and demographic covariates by using the multiple imputation approach developed by Rubin. ${ }^{42}$ As a sensitivity analysis, we carried out a complete case analysis, and this gave similar results.

We calculated means and SD for quantitative variables, and frequencies and proportions for categorical ones. Univariate and multivariate linear regressions were carried out to identify variables of HL. Using sensitivity analysis, we checked whether a model without imputations would produce the same results as the model with multiple imputations: analysis with and without imputation gave similar results.
All analyses were performed using R software V.3.3.2 $2^{43}$ and the MICE package V.2.29. ${ }^{44}$

\section{RESULTS}

\section{Descriptive analyses}

Cases with at least one missing value among the six items were considered incomplete (239, 18, 417, 168, 252 and 175 missing values for questions (1) to (6), respectively). Of the original 888 patients, 200, 175, 117, 59, 24 and 2 had, respectively, 1, 2, 3, 4, 5 and 6 missing HL variables. Thus, a complete HL score was only computable for 311 respondents (35\%), and we therefore chose to impute the missing values.

The mean age (SD) of the participants was 72.9 (12.0) years old; $52.0 \%$ were women. Almost half of the participants were married $(49.0 \%)$, and $40.0 \%$ had a tertiary level of education. The mean (SD) number of chronic conditions was 7.20 (2.9), and the mean (SD) number of medical visits in the last year was 12.90 (8.7). The mean HL score (SD) of the participants was 2.9 (0.5). Descriptive statistics are summarised in table 1.

In bivariate analyses with imputation, we found significant relationships between HL and almost all the covariates except for the number of chronic conditions, number of drugs, number of medical visits in the last year, number of hospitalisations in the last year, homecare, nurse and SI (table 2).

The multivariate analyses showed significant relationships between HL and the treatment burden score $(\beta=-0.004,95 \%$ CI -0.006 to 0.002$)$; marital status, but particularly for the divorced group $(\beta=0.136,95 \% \mathrm{CI}$ 0.012 to 0.260 ); dimensions of the EQ5D3L quality of life assessment, that is, moderate problems with mobility and moderate and severe problems with anxiety/depression ( $\beta=-0.086,95 \% \mathrm{CI}-0.157$ to 0.016$),(\beta=-0.129,95 \% \mathrm{CI}$ -0.198 to 0.060 and $\beta=-0.343,95 \%$ CI -0.500 to 0.186 ), respectively. Table 3 shows the results of these multivariate analyses.

\section{DISCUSSION}

The present study showed that the mean (SD) HL score in our multimorbid primary care patient sample was 2.9 (0.5). In multimorbid patients, a high treatment burden and effects on patients' quality of life due to problems with mobility and anxiety/depression were negatively associated with HL. However, our study revealed no association between HL and age. Although several studies have assessed HL, to the best of our knowledge, little is known about which factors are associated with low HL in multimorbid patients in primary care.

The present study's main finding was that the treatment burden facing multimorbid primary care patients was negatively associated with HL. In other words, the lower a multimorbid patient's HL, the higher the treatment burden. This is a very interesting finding, and although the $\beta$ coefficient is small, we believe that this result is 
Table 1 Descriptive statistics of the sample of multimorbid patients, $\mathrm{n}=888$

\begin{tabular}{lll}
\hline Variable & Mean* $^{*}$ & SD* $^{*}$ \\
\hline Age & 72.93 & 12.00 \\
$\begin{array}{l}\text { Health literacy score (577 missing } \\
\text { values) }\end{array}$ & 2.87 & 0.45
\end{tabular}

\begin{tabular}{|lll|}
\hline Material deprivation score & 0.50 & 1.27 \\
\hline Social deprivation score & 1.93 & 1.38 \\
\hline Health deprivation Score & 0.48 & 0.68 \\
\hline No of medical visits last year & 12.90 & 8.70
\end{tabular}

(one missing value)

\begin{tabular}{|c|c|c|}
\hline $\begin{array}{l}\text { No of hospitalisations } \\
\text { (three missing values) }\end{array}$ & 0.54 & 0.99 \\
\hline $\begin{array}{l}\text { No of chronic conditions } \\
\text { (four missing values) }\end{array}$ & 7.20 & 2.86 \\
\hline TBQ score & 26.77 & 18.60 \\
\hline No of specialists (141 missing values) & 2.23 & 1.27 \\
\hline Severity Index & 1.75 & 0.38 \\
\hline Visual Analogue Scale (EQ5D3L) & 63.19 & 19.25 \\
\hline Variable & Count & Prop \\
\hline \multicolumn{3}{|l|}{ Sex } \\
\hline Male & 428 & 0.48 \\
\hline Female & 460 & 0.52 \\
\hline \multicolumn{3}{|l|}{ Educational level (one missing value) } \\
\hline Primary & 195 & 0.22 \\
\hline Secondary & 337 & 0.38 \\
\hline Tertiary & 355 & 0.40 \\
\hline \multicolumn{3}{|l|}{ Marital status } \\
\hline Single & 85 & 0.10 \\
\hline Married & 437 & 0.49 \\
\hline Divorced & 150 & 0.17 \\
\hline Widowed & 216 & 0.24 \\
\hline \multicolumn{3}{|l|}{ No of drugs } \\
\hline $0-4$ & 156 & 0.18 \\
\hline $5-6$ & 212 & 0.24 \\
\hline $7-9$ & 276 & 0.31 \\
\hline$\geq 10$ & 244 & 0.27 \\
\hline \multicolumn{3}{|l|}{ Home nurse (one missing value) } \\
\hline No & 798 & 0.90 \\
\hline Yes & 89 & 0.10 \\
\hline
\end{tabular}

Paramedical† (12 missing values)

\begin{tabular}{|c|c|c|}
\hline No & 572 & 0.65 \\
\hline Yes & 304 & 0.35 \\
\hline \multicolumn{3}{|c|}{ Homecare (one missing value) } \\
\hline No & 755 & 0.85 \\
\hline Yes & 132 & 0.15 \\
\hline
\end{tabular}

EQ5D3L five dimensions

Mobility

\begin{tabular}{lll}
\hline Table 1 Continued & & \\
\hline Variable & Mean* & SD* $^{*}$ \\
\hline No problems & 497 & 0.56 \\
Moderate problems & 386 & 0.43 \\
$\quad$ Severe problems & 5 & 0.01 \\
Self-care & & \\
$\quad$ No problems & 785 & 0.88 \\
Moderate problems & 92 & 0.10 \\
$\quad$ Severe problems & 11 & 0.01 \\
Usual activities & & \\
No problems & 543 & 0.61 \\
Moderate problems & 328 & 0.37 \\
$\quad$ Severe problems & 17 & 0.02 \\
Pain/discomfort & & \\
No problems & 211 & 0.24 \\
Moderate problems & 591 & 0.67 \\
Severe problems & 86 & 0.10 \\
Anxiety/depression & & \\
No problems & 516 & 0.58 \\
Moderate problems & 329 & 0.37 \\
Severe problems & 43 & 0.05 \\
\hline
\end{tabular}

*With multiple imputation.

†Paramedical includes physiotherapists/occupational therapists. EQ5D3L, EuroQuol 5 Dimension 3 Level; TBQ, Treatment Burden Questionnaire.

clinically relevant and allows us to identify treatment burden as an element to take into account for potentially low literacy in multimorbid patients.

There are no specific references to explain the association found between HL and the burden of treatment. As a proxy for treatment burden, some authors have described an association between low HL and treatment adherence $^{45}{ }^{46}$; however, others found no evidence for such an association. ${ }^{47} 48$ Our exploratory study was unable to determine any causal association between the treatment burden and HL. But our results could also be considered from another angle, that has been taken in other analyses. Indeed, individuals with low HL are usually less well educated and belong to lower socioeconomic groups. ${ }^{18}$ Multimorbidity is higher in these groups and patients in them are likely to suffer more severely and from more diseases, leading to a higher treatment burden. Furthermore, low HL, used as an independent variable, has been found to be associated with a reduced ability of adults with coronary heart disease to identify medication, ${ }^{49}$ poor health outcomes ${ }^{14} 1950$ and a more significant use of healthcare. ${ }^{51}$ These three factors can also be linked to multimorbidity and may have an impact on the overall treatment burden.

Another important finding was that patients whose quality of life had been altered by problems related to anxiety/depression or mobility were more likely to have 
Table 2 Results of bivariate analyses with multiple imputation

\begin{tabular}{|c|c|c|c|}
\hline Independent variable & $\beta$ & $95 \% \mathrm{Cl}$ & $P$ value \\
\hline Age & 0.0053 & 0.0027 to 0.0079 & 0.0001 \\
\hline Sex & -0.1008 & -0.1643 to 0.0372 & 0.0019 \\
\hline Material deprivation & -0.0786 & -0.1034 to 0.0537 & 0.0000 \\
\hline Social deprivation & -0.0485 & -0.0707 to 0.0263 & 0.0000 \\
\hline \multicolumn{4}{|l|}{ Educational level (reference: primary) } \\
\hline Secondary & 0.0408 & -0.0464 to 0.1280 & 0.3579 \\
\hline Tertiary & 0.0880 & 0.0034 to 0.1726 & 0.0415 \\
\hline No of medical visits last year & -0.0023 & -0.0060 to 0.0014 & 0.2285 \\
\hline No of hospitalisations & -0.0283 & -0.0598 to 0.0033 & 0.0789 \\
\hline $7-9$ & 0.0025 & -0.0926 to 0.0976 & 0.9588 \\
\hline$\geq 10$ & -0.0601 & -0.1579 to 0.0377 & 0.2279 \\
\hline TBQ score & -0.0071 & -0.0086 to 0.0055 & 0.0000 \\
\hline Home nurse & -0.1016 & -0.2133 to 0.0100 & 0.0743 \\
\hline Paramedical $^{*}$ & -0.0843 & -0.1489 to 0.0198 & 0.0105 \\
\hline Homecare & -0.0772 & -0.1698 to 0.0153 & 0.1016 \\
\hline No of specialists & -0.0284 & -0.0544 to 0.0024 & 0.0322 \\
\hline Severity Index & -0.0457 & -0.1258 to 0.0344 & 0.2630 \\
\hline Visual Analogue Scale (EQ5D3L) & 0.0048 & 0.0032 to 0.0064 & 0.0000 \\
\hline Moderate problems & -0.1965 & -0.3045 to 0.0884 & 0.0004 \\
\hline Severe problems & -0.1464 & -0.4724 to 0.1795 & 0.3755 \\
\hline \multicolumn{4}{|c|}{ Usual activities (reference: no problems) } \\
\hline Moderate problems & -0.1729 & -0.2371 to 0.1086 & 0.0000 \\
\hline Severe problems & -0.4167 & -0.6521 to 0.1813 & 0.0006 \\
\hline \multicolumn{4}{|c|}{ Pain/discomfort (reference: no problems) } \\
\hline Moderate problems & -0.1174 & -0.1970 to 0.0378 & 0.0040 \\
\hline Severe problems & -0.2760 & -0.3961 to 0.1558 & 0.0000 \\
\hline \multicolumn{4}{|c|}{ Anxiety/depression (reference: no problems) } \\
\hline Moderate problems & -0.2211 & -0.2839 to 0.1584 & 0.0000 \\
\hline Severe problems & -0.5390 & -0.6788 to 0.3991 & 0.0000 \\
\hline \multicolumn{4}{|l|}{ Marital status (reference: single) } \\
\hline Married & 0.1596 & 0.0484 to 0.2709 & 0.0050 \\
\hline Divorced & 0.0947 & -0.0315 to 0.2208 & 0.1412 \\
\hline Widowed & 0.1128 & -0.0057 to 0.2313 & 0.0619 \\
\hline
\end{tabular}

*Paramedical includes physiotherapists/occupational therapists.

EQ5D3L, EuroQuol 5 Dimension 3 Level; TBQ, Treatment Burden Questionnaire. 
Table 3 Results of multivariate analyses with multiple imputation

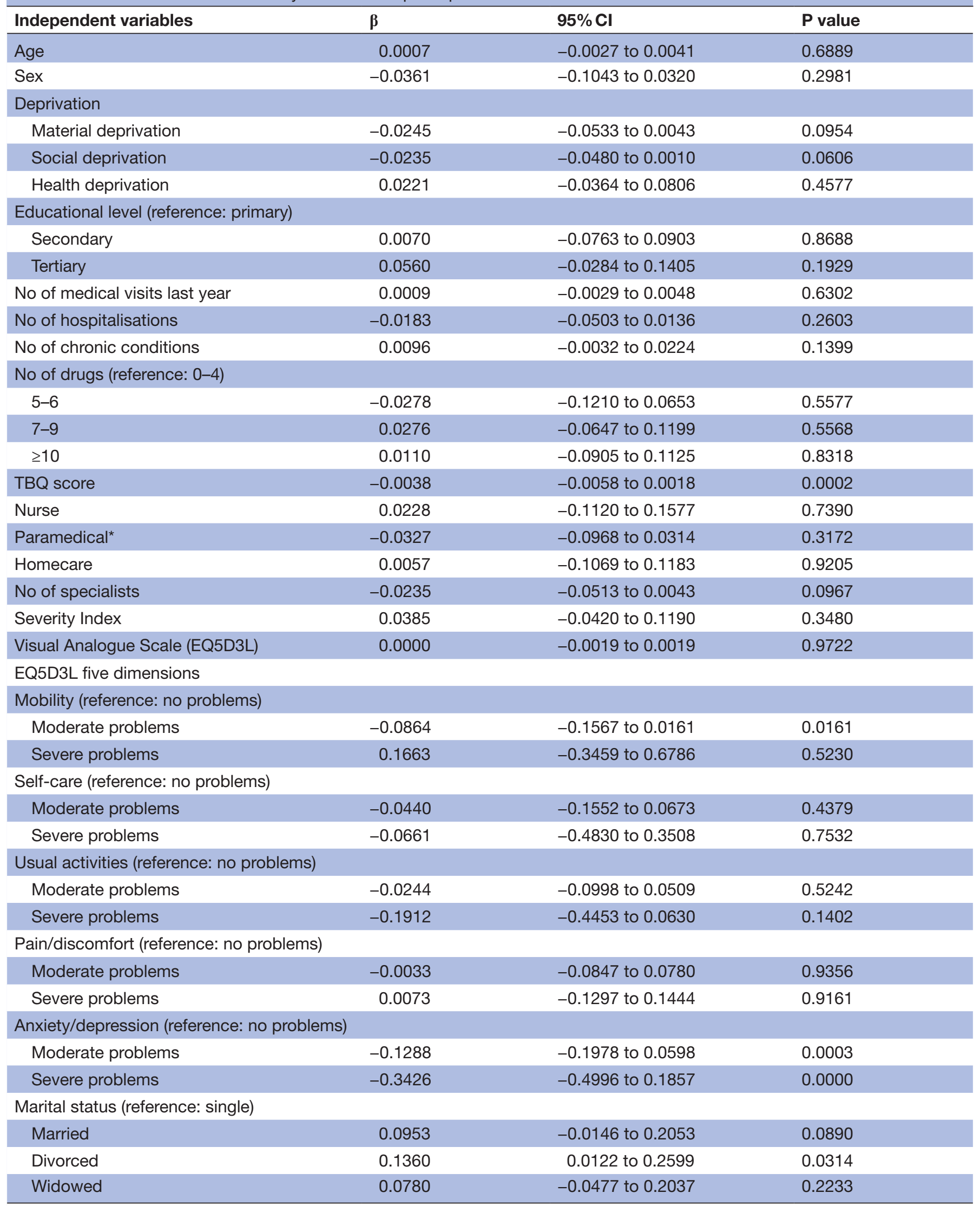

${ }^{*}$ Paramedical includes physiotherapists/occupational therapists.

EQ5D3L, EuroQuol 5 Dimension 3 Level; TBQ, Treatment Burden Questionnaire. 
low levels of HL. As described by DiMatteo et al, anxiety and depression may affect patients' motivation and ability $^{52}$ to seek out and understand information about their diseases and treatment, resulting in a lower level of HL, especially in the complex context of multimorbidity. Moreover, anxiety and depression may well be higher when patients have to face up to more numerous health problems, especially in multimorbidity. This result is not concordant with the study by Green $e t a l,{ }^{53}$ which found no association between limited HL and depression in patients receiving chronic haemodialysis treatment, but this difference might be explained by the differences in the study populations, how HL was assessed and the fact that his study used HL as independent variable.

Concerning the association between mobility problems and low HL, Matsumoto and Nakayama describe a relationship with different social determinants of health such as housing, employment, transport or social support. ${ }^{54}$ On the other hand, in a very different population, there was a strong association between low HL and obesity in young children with a described lack of physical activity. ${ }^{55}$ However, we can only speculate as to whether there is any precise explanation of the associations between anxiety, depression, mobility and HL. Furthermore, due to its design, the study's findings cannot determine a causal association.

Anxiety, depression and mobility are just some of the determinants of quality of life, and the literature reveals very controversial results concerning the association between HL and quality of life. It seems that such associations may depend on certain other aspects, such as specific chronic diseases or the cultural characteristics of the population studied. ${ }^{56}$ Thus, although we found that some of the determinants of quality of life are factors associated with low HL, we believe that further studies are necessary to better understand the underlying reasons for the controversial results in the literature. However, it nevertheless seems important to describe factors associated with low HL, thus allowing GPs to better identify such patients and to adapt how they inform them about medical problems.

The present study found no association between the level of HL of multimorbid primary care patients and their use of healthcare services, especially with regards to the number of consultations with their GP or the number of hospitalisations in the past year. Results in the literature are inconsistent. The study by Vandenbosch $e t$ $a \iota^{51}$ found no significant associations between HL and the number of medical visits or hospitalisations, whereas a study by Duong et $a \tilde{l}^{\tilde{7}}$ described an association between HL and healthcare use. These results are inconsistent due to differences in the study populations (ie, our study population was composed of multimorbid patients in primary care settings) and methodologies (other studies considered HL as an independent variable). Indeed, we suggest that HL in multimorbid patients is different from that in the general population and should be assessed differently. Further research should aim to clearly confirm or invalidate our results with multimorbid patients.

\section{Strengths and limitations}

This national primary care study enabled the analysis of data from a relatively representative sample of multimorbid patients suffering from at least three chronic conditions and enrolled in GPs' practices across Switzerland. Although several studies have previously assessed $\mathrm{HL}$, to the best of our knowledge, this was the first to have assessed factors associated with HL in multimorbid patients in a primary care setting.

However, this study had some limitations. First, the $\beta$ coefficients were small, making clinical interpretation difficult, despite the fact that the study gives an overall view of the factors associated with HL. Further studies should be done to confirm these trends.

Second, our sample might not be perfectly representative of all multimorbid patients. GPs only recruited patients who came to their practices and who were suffering from at least 3 chronic conditions from a list of 75 provided to them. The most impaired multimorbid patients, therefore, those with the most extensive mobility problems (ie, those cared for via homecare visits, in nursing homes or hospitalised) were not included (selection bias). Our results concerning HL in multimorbid patients should thus be interpreted with caution, taking into consideration multimorbid patients who cannot attend GPs' practices or who have rare chronic conditions. Furthermore, we cannot exclude a potential selection bias, as patients who consulted more frequently had a higher chance of being included. However, we found no association between the frequency of consultations and HL in our final model.

Third, the HL score contained a lot of missing values, and we cannot exclude that the cause of that missing data was related to HL itself. However, we examined whether the mean of the available HL items was associated with the number of missing HL items, and this was not the case.

Fourth, due to the study's cross-sectional design, causal relationships cannot be inferred.

\section{CONCLUSION}

The present study highlights factors associated with HL in multimorbid patients in primary care: a high treatment burden, altered quality of life by problems with anxiety or depression and poor mobility were associated with a low level of HL. This is a useful information that could guide GPs in their daily practice and help them to better identify patients at risk of having low HL. Even though, with the current state of knowledge, we cannot demonstrate causal relationships between multimorbidity and the treatment burden, GPs should carefully weigh up how best to transmit clinical information to patients whom they believe to be at risk of low HL. 
Acknowledgements The authors are grateful to all the GPs and patients for their extensive participation.

Contributors AAN, AD-L, BB, DMH, AZ, LH and PB developed the protocol for the MMFM study. LH, AZ and DMH ensured the recruitment of participating GPs. AAN was primarily responsible for planning the analysis and drafting the manuscript. JP was responsible for the analyses. $\mathrm{PB}, \mathrm{JP}$, and $\mathrm{LH}$ helped to improve the manuscript during this process. $\mathrm{BB}, \mathrm{AD}, \mathrm{DMH}, \mathrm{SS}, \mathrm{SNJ}, \mathrm{AZ}, \mathrm{PB}$ and $\mathrm{LH}$ made contributions to the interpretation of the findings and the content of the final manuscript. All authors read and approved the final manuscript.

Funding This work was supported by Swiss University Conference project P10, which granted funding to reinforce teaching and research in Primary Care in Switzerland. Within the framework of this financial support, Switzerland's five university institutes of family medicine (in Basel, Bern, Geneva, Lausanne and Zurich) collaborate as the Swiss Academy of Family Medicine (SAFMED).

Disclaimer The funding source had no role in study design, data collection, analysis or interpretation, nor in the preparation of the manuscript and the decision to submit the paper for publication.

Competing interests None declared.

Patient consent Obtained.

Ethics approval The study protocol (Protocol No 314/15) was approved by the Human Research Ethics Committee of the Canton Vaud, Switzerland.

Provenance and peer review Not commissioned; externally peer reviewed.

Data sharing statement Data are available at family medicine institute of Lausanne.

Open Access This is an Open Access article distributed in accordance with the Creative Commons Attribution Non Commercial (CC BY-NC 4.0) license, which permits others to distribute, remix, adapt, build upon this work non-commercially, and license their derivative works on different terms, provided the original work is properly cited and the use is non-commercial. See: http://creativecommons.org/ licenses/by-nc/4.0/

(c) Article author(s) (or their employer(s) unless otherwise stated in the text of the article) 2018. All rights reserved. No commercial use is permitted unless otherwise expressly granted.

\section{REFERENCES}

1. Uijen $A A$, van de Lisdonk EH. Multimorbidity in primary care: prevalence and trend over the last 20 years. Eur $J$ Gen Pract 2008;14(Suppl 1):28-32.

2. Valderas JM, Starfield B, Sibbald B, et al. Defining comorbidity: implications for understanding health and health services. Ann Fam Med 2009:7:357-63.

3. van den Akker M, Buntinx F, Metsemakers JF, et al. Multimorbidity in general practice: prevalence, incidence, and determinants of co-occurring chronic and recurrent diseases. J Clin Epidemiol 1998;51:367-75.

4. Goodman RA, Posner SF, Huang ES, et al. Defining and measuring chronic conditions: imperatives for research, policy, program, and practice. Prev Chronic Dis 2013;10:10.

5. Heyworth IT, Hazell ML, Linehan MF, et al. How do common chronic conditions affect health-related quality of life? Br J Gen Pract 2009;59:e353-8.

6. Condelius A, Edberg AK, Jakobsson U, et al. Hospital admissions among people 65+ related to multimorbidity, municipal and outpatient care. Arch Gerontol Geriatr 2008;46:41-55.

7. Smith SM, O'Dowd T. Chronic diseases: what happens when they come in multiples? Br J Gen Pract 2007;57:268-70.

8. Vogeli $\mathrm{C}$, Shields AE, Lee TA, et al. Multiple chronic conditions: prevalence, health consequences, and implications for quality, care management, and costs. J Gen Intern Med 2007;22 (Suppl 3):391-5.

9. Wehling M, Multimorbidity WM. Multimorbidity and polypharmacy: how to reduce the harmful drug load and yet add needed drugs in the elderly? Proposal of a new drug classification: fit for the aged. $J$ Am Geriatr Soc 2009;57:560-1.

10. Bayliss EA, Edwards AE, Steiner JF, et al. Processes of care desired by elderly patients with multimorbidities. Fam Pract 2008;25:287-93.

11. Bodenheimer T, Fernandez A. High and rising health care costs. Part 4: can costs be controlled while preserving quality? Ann Intern Med 2005;143:26-31.
12. Taylor AW, Price K, Gill TK, et al. Multimorbidity - not just an older person's issue. Results from an Australian biomedical study. BMC Public Health 2010;10:718.

13. Kindig DA, Panzer AM, Nielsen-Bohlman L. Health literacy: a prescription to end confusion: National Academies Press. 2004

14. Hudon C, Fortin M, Poitras ME, et al. The relationship between literacy and multimorbidity in a primary care setting. BMC Fam Pract 2012;13:33

15. Hersh L, Salzman B, Snyderman D. Health literacy in primary care practice. Am Fam Physician 2015;92:92.

16. Bennett IM, Chen J, Soroui JS, et al. The contribution of health literacy to disparities in self-rated health status and preventive health behaviors in older adults. Ann Fam Med 2009;7:204-11.

17. van der Heide I, Uiters E, Sørensen K, et al. Health literacy in Europe: the development and validation of health literacy prediction models. Eur J Public Health 2016;26:906-11.

18. van der Heide I, Wang J, Droomers M, et al. The relationship between health, education, and health literacy: results from the Dutch Adult Literacy and Life Skills Survey. J Health Commun 2013;18 (Suppl 1):172-84.

19. Berkman ND, Sheridan SL, Donahue KE, et al. Low health literacy and health outcomes: an updated systematic review. Ann Intern Med 2011:155:97-107.

20. Friis K, Lasgaard M, Osborne $\mathrm{RH}$, et al. Gaps in understanding health and engagement with healthcare providers across common long-term conditions: a population survey of health literacy in 29,473 Danish citizens. BMJ Open 2016;6:e009627.

21. Barrett SE, Puryear JS. Health literacy: improving quality of care in primary care settings. J Health Care Poor Underserved 2006;17:690-7.

22. Al Sayah F, Majumdar SR, Williams B, et al. Health literacy and health outcomes in diabetes: a systematic review. J Gen Intern Med 2013;28:444-52.

23. Seligman HK, Wang FF, Palacios JL, et al. Physician notification of their diabetes patients' limited health literacy: A randomized, controlled trial. J Gen Intern Med 2005;20:1077.

24. Smith SK, Nutbeam D, McCaffery KJ. Insights into the concept and measurement of health literacy from a study of shared decision-making in a low literacy population. J Health Psychol 2013;18:1011-22.

25. Smith SG, Curtis LM, Wardle J, et al. Skill set or mind set? Associations between health literacy, patient activation and health. PLoS One 2013:8:e74373.

26. Durand MA, Carpenter L, Dolan H, et al. Do interventions designed to support shared decision-making reduce health inequalities? A systematic review and meta-analysis. PLoS One 2014;9:e94670.

27. Quenzel G, Schaeffer D, Messer M, et al. [Health literacy among less well-educated young people: Influencing factors and consequences] Bundesgesundheitsblatt Gesundheitsforschung Gesundheitsschutz 2015;58:951-7.

28. Sørensen K, Pelikan JM, Röthlin F, et al. Health literacy in Europe: comparative results of the European health literacy survey (HLS-EU). Eur J Public Health 2015;25:1053-8.

29. Déruaz-Luyet $A, N^{\prime}$ Goran $A A$, Tandjung $R$, et al. Multimorbidity in primary care: protocol of a national cross-sectional study in Switzerland. BMJ Open 2015;5:e009165.

30. Déruaz-Luyet A, N'Goran AA, Senn N, et al. Multimorbidity and patterns of chronic conditions in a primary care population in Switzerland: a cross-sectional study. BMJ Open 2017;7:e013664.

31. N'Goran AA, Blaser J, Deruaz-Luyet A, et al. From chronic conditions to relevance in multimorbidity: a four-step study in family medicine. Fam Pract 2016;33:439-44. cmw030.

32. Hofmans-Okkes IM, Lamberts H. The International Classification of Primary Care (ICPC): new applications in research and computer-based patient records in family practice. Fam Pract 1996;13:294-302.

33. Consortium H-E. Comparative report of health literacy in eight EU member states. The European health literacy survey HLS-EU 2012

34 Pelikan J, Ganahl K, Röthlin F, et al. Measuring comprehensive health literacy in general populations - le HLS-EU instruments. Presentation at the Second International Conference of Health Literacy and Health Promotion. 2014.

35. Sørensen K, Van den Broucke S, Pelikan JM, et al. Measuring health literacy in populations: illuminating the design and development process of the European Health Literacy Survey Questionnaire (HLSEU-Q). BMC Public Health 2013;13:948.

36. Brett T, Arnold-Reed DE, Popescu A, et al. Multimorbidity in patients attending 2 Australian primary care practices. Ann Fam Med 2013;11:535-42.

37. Miller M, Towers A. A manual of guidelines for scoring the Cumulative IIIness Rating Scale for Geriatrics (CIRS-G). Pittsburgh, PA: University of Pittsburgh, 1991. 
38. Miller MD, Paradis CF, Houck PR, et al. Rating chronic medical illness burden in geropsychiatric practice and research: application of the Cumulative IIIness Rating Scale. Psychiatry Res 1992;41:237-48.

39. Linn BS, Linn MW, Gurel L. Cumulative illness rating scale. J Am Geriatr Soc 1968;16:622-6.

40. Vaucher P, Bischoff T, Diserens EA, et al. Detecting and measuring deprivation in primary care: development, reliability and validity of a self-reported questionnaire: the DiPCare-Q. BMJ Open 2012;2:e000692.

41. Tran VT, Montori VM, Eton DT, et al. Development and description of measurement properties of an instrument to assess treatment burden among patients with multiple chronic conditions. BMC Med 2012;10:68.

42. Rubin R. Multiple imputation for nonresponse in surveys. New York, NY: Wiley \& Sons, 1987.

43. RCore T. R: A language and environment for statistical computing. Vienna, Austria: R Foundation for Statistical Computing, 2013. http:// www R-project org

44. Buuren Svan, Groothuis-Oudshoorn K. mice : Multivariate Imputation by Chained Equations in R. J Stat Softw 2011;45.

45. Kalichman SC, Ramachandran B, Catz S. Adherence to combination antiretroviral therapies in HIV patients of low health literacy. J Gen Intern Med 1999;14:267-73.

46. Muir KW, Santiago-Turla C, Stinnett SS, et al. Health literacy and adherence to glaucoma therapy. Am J Ophthalmol 2006;142:223-6.

47. Loke YK, Hinz I, Wang $X$, et al. Systematic review of consistency between adherence to cardiovascular or diabetes medication and health literacy in older adults. Ann Pharmacother 2012;46:863-72.

48. Baruel Okumura PC, Okumura LM, Reis WC, et al. Comparing medication adherence tools scores and number of controlled diseases among low literacy patients discharged from a Brazilian cardiology ward. Int J Clin Pharm 2016;38:1362-6.

49. Kripalani S, Henderson LE, Chiu EY, et al. Predictors of medication self-management skill in a low-literacy population. J Gen Intern Med 2006;21:852-6.

50. Baker DW, Parker RM, Williams MV, et al. The relationship of patient reading ability to self-reported health and use of health services. Am J Public Health 1997;87:1027-30.

51. Vandenbosch J, Van den Broucke S, Vancorenland S, et al. Health literacy and the use of healthcare services in Belgium. J Epidemiol Community Health 2016;70:1032-8.

52. DiMatteo MR, Lepper HS, Croghan TW. Depression is a risk factor for noncompliance with medical treatment: meta-analysis of the effects of anxiety and depression on patient adherence. Arch Intern Med 2000;160:2101-7.

53. Green JA, Mor MK, Shields AM, et al. Prevalence and demographic and clinical associations of health literacy in patients on maintenance hemodialysis. Clin J Am Soc Nephrol 2011;6:1354-60.

54. Matsumoto M, Nakayama K. Development of the health literacy on social determinants of health questionnaire in Japanese adults. BMC Public Health 2017:17:30.

55. Shih SF, Liu CH, Liao LL, et al. Health literacy and the determinants of obesity: a population-based survey of sixth grade school children in Taiwan. BMC Public Health 2016;16:280.

56. Couture ÉM, Chouinard MC, Fortin M, et al. The relationship between health literacy and quality of life among frequent users of health care services: a cross-sectional study. Health Qual Life Outcomes 2017;15:137.

57. Duong VT, Lin IF, Sorensen K, et al. Health literacy in Taiwan: a population-based study. Asia Pac J Public Health 2015;27:871-80. 